\title{
CONTRIBUTIONS AND ELECTIONS WITH NETWORK EXTERNALITIES
}

\author{
Adam Meirowitz and Alan E. Wiseman*
}

\begin{abstract}
This paper develops a model of campaign contributions and electoral competition. Contributors have separable preferences over policy and the electoral success of the candidate they support, as in influence buying. Policy preferences are single peaked over a single policy dimension. A candidate's chances of victory are increasing in the relative size of her war chest. In equilibrium, potential contributors balance incentives to donate to a candidate that is desirable on policy grounds and ensuring that they back the likely winner. With exogenous candidate positions, we find conditions under which, in equilibrium, contributors donate to the candidate that is less desirable on policy grounds solely because they consider the candidate viable. We also find that there is a degree of indeterminacy, wherein multiple equilibria inducing different lotteries over the final policy often exist. With endogenous candidate locations, we find that while median policies are always supportable as equilibrium, it is often the case that any pair of candidate locations is supportable in equilibrium. These results suggest that in settings with substantial influence buying, median policy interests may not be represented.
\end{abstract}

There's nothing I hate more than being at a cocktail party and having someone come up to me and say: "I think your boss is awesome, and I wish that he'd win, but there's no way I'm going to contribute to him ... he can't overcome Bush's money machine."

(Elizabeth Chenoweth, fundraiser for McCain2000, Summer 1999)

IN MARCH 1999, George W. Bush sent out a one-page letter announcing the formation of his "Presidential Exploratory Committee." This initial letter, which included a brief solicitation for campaign funds, led to the quick generation of a \$7.6 million war chest within four weeks (Van Natta, 2000). As 1999 went on, the war chest grew at a spectacular rate. At the end of second quarter 1999, the campaign had raised nearly \$37 million and had \$30 million cash on hand; and as 1999 closed out, the Bush campaign had raised well over $\$ 60$ million. While pundits and casual observers watched in awe as the war chest grew, potential competitors watched their campaigns quickly flounder as they were unable to secure the necessary funds for survival. In September, Dan Quayle withdrew from the race, noting that he was

\footnotetext{
*Contact address: Alan E. Wiseman, Department of Political Science, The Ohio State University, 2140 Derby Hall, 154 North Oval Mall, Columbus, OH 43210-1373, USA. E-mail: wiseman.69@osu.edu
} 
"facing a campaign where the front-runner would have up to $\$ 100$ million to spend" (Glasser, 1999). Later that Fall, Elizabeth Dole argued in midOctober that "it's not all just money ... it's experience and the people you bring into the party" (Wilkie, 1999). Despite her passionate pleas for expanding the Republican base, she left the contest only a few days later, stating that "the bottom line remains money - it restricts your ability to communicate with voters ... it would be futile to continue" (Miga, 1999).

While it is obvious that the great disparity between Bush's funds and these candidates' war chests led them to drop out, one looks at the campaign dynamics of the 2000 primaries and wonders how, precisely, did Bush manage to generate such a massive amount of money? Make no mistake, not only was Bush's treasury growing at an astounding rate, but the sheer magnitude of his purse dwarfed all preceding contests. In April of 1996, Clinton and Dole had raised about $\$ 39$ million and $\$ 41$ million, respectively. As of May 2000, Bush had raised more than $\$ 82$ million for his cause. ${ }^{1}$ Is it truly the case that there were so many people who felt that Bush was such an outstanding candidate that they came out in droves with financial support? Or is it possibly the case that many people found themselves giving to the campaign primarily because of the expectation that other donors were doing the same, leading to a self-fulfilling prophecy of an impregnable war chest?

Moving past the campaign, the Bush-Gore race was as close to a tie as we have seen in recent history. When all was said and done, the final tally was a statistical dead heat, with Gore only barely beating Bush in the popular vote. From a purely spatial perspective, this should be unsurprising: most observers (and Ralph Nader) would argue that both candidates had become virtually identical on many policy dimensions, and hence it would be expected for them to split the vote down the middle. This outcome may seem at odds with the fundraising phenomena that characterized the general election. As of August 2000, Bush had raised almost $\$ 95$ million compared to Gore's \$49 million war chest. $^{2}$ This disparity existed despite the fact that throughout much of the summer, Gore was picked to be the sure winner. After all, he was an incumbent vice president for a president with high approval ratings during one of the most prosperous economic periods during the twentieth century. That being said, why was there such a monstrous resource disparity? If these candidates looked the same, why would donors be giving to them at different rates? And furthermore, why would so many contributors be giving to a candidate that most pundits had picked to be the underdog?

This paper addresses these issues by developing a model of campaign contributions with network externalities. Potential donors are faced with a decision of who to give their money to. Contributors base their decision on personal policy preferences and expectations about the behavior of other potential

\footnotetext{
${ }^{1}$ Source: http://www.fec.gov

${ }^{2}$ Source: http://www.fec.gov/finance/precm8.htm
} 
donors. We assume that the policy space is unidimensional, that contributor policy preferences are single peaked, and that contributors prefer donating to the winning candidate rather than the losing candidate. In a model in which candidate locations are exogenous, results identify cases in which a large portion of contributors may support an undesirable candidate simply because a majority of contributors have chosen to support that candidate. Such cases can be socially inefficient in that a dominant candidate might emerge who is less favorable (from a policy preference standpoint) to a majority of the electorate than his competitor. In addition, sometimes there are multiple self-sustaining beliefs about how the population of contributors will act. This suggests that the presence of money in campaigns may result in equilibrium candidate locations that are not closely related to the policy preferences of the electorate.

The indeterminacy in the exogenous policy platform model is fundamentally the product of the assumption that contributors value giving to the winning candidate. The existence of multiple equilibria in models of this form is quite similar to work on technology adoption and network externalities in which consumers care both about product characteristics and the likelihood that one product will become a dominant standard.

In the model in which candidate locations are endogenous, the equilibrium analysis is more complicated. An equilibrium in this model requires that candidates form beliefs over which equilibria will occur in off-the-equilibriumpath histories (policy platform pairs). Since there is no natural way to limit these beliefs, the equilibrium set is often large. It is always possible to support simultaneous selection of the median voter's ideal point as an equilibrium. In this equilibrium, the ex-ante favored candidate receives fewer contributions, and contributions are uncorrelated with the probability that a candidate wins. This equilibrium may approximate the Bush-Gore race where Gore was the favored candidate in the summer, Bush dramatically outspent Gore, and the election was a tie. While this median-convergence equilibrium seems focal, it is not unique. In fact, for a large class of parameterizations, any profile of candidate stances can occur in an equilibrium.

The paper is organized as follows. Section 1 discusses the relevant literature on contribution behavior as well as introducing some of the central concepts in the body of economic research that deals with network externalities. Section 2 develops a campaign contribution game and section 3 presents the equilibrium results of the game. Section 4 presents the larger candidate location game and the analysis of this game. Finally, section 5 concludes with a discussion of further theoretical and empirical extensions to this research project. Proofs of all results appear in the Appendix.

\section{PREVIOUS RESEARCH}

While there has been a substantial body of empirical research investigating the role and effects of money in elections [e.g. Dunn (1972); Jacobsen (1980); 
Sorauf $(1988,1992)]$, there has been less empirical scholarship focusing on the behavioral motivations behind individual donors. Analyzing surveys from contributors in the 1988 and 1992 election cycles, Brown et al. $(1995$, p. 46) find that many contributors donate because they believe that they can influence the outcome of the election or future public policies in some way. Francia et al. (1999, p. 134) find similar results when looking at the motives behind donors in the 1996 presidential election cycle. Over $70 \%$ of the contributors cited the prospect of influencing the outcome and/or future policies as a "very important" reason for giving. While this finding seems pervasive, one wonders whether it is truly rational. Current Federal Election Commission regulations limit individual contributions to $\$ 2,000$ per election, so it seems questionable whether any one contribution can affect the outcome of the race. ${ }^{3}$ Furthermore, while it is true that the lion's share of presidential candidates' war chests come from individual contributors, with so many people giving, the chances that any one individual, or even a small group of individuals, can exert any influence on enacted policy seems very low. ${ }^{4}$

In contrast to the findings of Brown et al. and other scholars, several theoretical studies (e.g. Baron, 1994; Snyder, 1990) have postulated that donors contribute to candidates so that they might receive particularistic treatment from the candidates if they achieve office. Hence, contributors are not motivated by any policy preferences per se, but rather the distributive benefits that might accrue to them if the candidate that they support wins the election. In recent and closely related work, Morton and Myerson (2000) embrace this notion of donors viewing their contributions as "investor-" or "serviceinduced" contributions, and assume that voters' preferences are defined over a candidate's policy stance and the amount of contributions he receives. Analyzing a "coordination" game among contributors, Morton and Myerson find that because all contributors wish to donate to a winner, the presence of any perceived advantage for one of the candidates can lead to all contributors donating to that candidate, which in turn leads to him becoming the expected victor. Morton and Myerson offer two broad conclusions:

First and foremost, our results imply that in elections in which campaign contributions are primarily given for private favors (investor contributions) and the electoral results are strongly influenced by such contributions, then the candidates involved have chosen convergent positions, close to that desired by the median voter. Thus, the existence of campaigns apparently driven by campaign spending may actually indicate electoral efficiency ... Finally, our

\footnotetext{
${ }^{3}$ The $\$ 2,000$ limit is a recent development, following from the passage of the McCain-Feingold campaign finance reform law. Prior to its passage, the contribution limit was $\$ 1,000$ for "hard" money. Even with such an increase in the limit, however, it seems reasonable to assert that any individual $\$ 2,000$ contribution will not change the electoral outcome.

${ }^{4}$ Brown et al. (1995, p. 6) note that individual contributions amounted to approximately $\$ 143$ million of presidential candidate campaign funds in the 1988 races, and \$82 million in the 1992 races.
} 
results suggest that incumbency advantages may simply reflect interest groups' use of incumbency as a focal point of coordination and that incumbents may have significant electoral advantages even when challengers offer not much of a difference in policy position from the incumbent [p. 26].

Our findings, while similar, have a different interpretation. While equilibria in which candidates locate close to the median ideal point exist, we also find that many other candidate profiles are supportable. Accordingly our analysis suggests that the presence of contributions may lead to electoral inefficiency. Second, by allowing for heterogeneity in the relationship between contributions and electoral advantages, our model allows us to consider elections in which the incumbent is viewed as advantaged by the voters. In such elections, when the race is close, it is the incumbent that receives fewer contributions. The logic is simple: if the race is close and the incumbent is advantaged she must be getting fewer contributions, otherwise she would be a more desirable candidate, would collect more contributions, and the race would not be close. The differences in findings in these two similar models are accounted for by two distinctions.

Morton and Myerson assume that voters care about ideology and advertisements (which are dependent on contributions) and that contributors are concerned only with the likelihood of success of the candidates. Alternatively, we assume contributors care both about policy and the electoral prospects of the candidates, and we allow voters to be primarily responsive to contributions. The model of voter choice in this study remains general, including models in which the pool of contributors and voters are identical. Throughout most of the analysis we assume that voter decisions are influenced only by contributions, but at the end of the analysis we demonstrate how the main results extend to a large class of models with voters that care about policy and contributions as long as neither candidate can win without a positive level of contributions. Instead of specifying the preferences of voters, per se, we consider a large class of mappings from contributions to votes. One advantage of this generality is that it allows us to consider various cases where one candidate is advantaged.

Baron's notion of informed and uninformed voters is useful in contrasting our study with Morton and Myerson. The latter assumes that a population of "investor" contributors is well-informed but does not care about policy. Thus, contributions are based exclusively on rational expectations about the electoral prospects of the candidates. Following contributions, uninformed voters that care about policy and are responsive to advertising/contributions vote. Our study allows for cases in which the roles are quite different. A population of well-informed contributors, concerned with both policy and the electoral prospects of candidates make contribution decisions. Following contributions, uninformed voters select candidates based (at least partially) on the advertising/contributions they observe. The motivational difference 
between the two studies is straightforward. Morton and Myerson's model of small contributions is motivated by the idea of influencing the electoral outcome for the purpose of receiving favors from the victor, whereas, in our model, contributors also care about the policy of the candidate they support.

This model draws on a large body of economics literature that focuses on commodities that possess "network externalities" or are subject to "network effects." A commodity or good is said to possess network externalities if the value of the product to a given user increases as more individuals use it. The most common example of such a product is a telephone system: with only one telephone user, the system is basically useless, but as more people start to own and use telephones, the value of the system increases immensely. Network externality models have traditionally focused on questions of technology adoption and/or industry standard setting [e.g. Farrell and Saloner (1985, 1986); Katz and Shapiro (1985, 1986); Rohlfs (1974)]. ${ }^{5}$ Given that consumers will choose particular products both as a function of their personal tastes and their expectations about future network size, scholars have tried to identify conditions that induce adoption of socially efficient standards, as well as tactics that competing firms might employ to ensure that their product becomes the dominant technology.

While not perfectly analogous, the campaign contribution decisions may be similar to cases in which consumers choose between products that exhibit network externalities. At any time, a given donor must choose who to give his money to, and this decision is likely shaped by his preferences over the candidates' stances as well as their chances of victory, given his beliefs about the contributions they will raise. Similar to these models of technology adoption, it is reasonable to expect that a socially inefficient candidate can emerge as the "dominant standard," in that the majority of donors contribute to him, despite the fact that they find him undesirable on policy grounds.

\section{MODEL}

\subsection{The Contribution Game}

We begin by describing a game in which a large population of contributors decide which of two candidates to provide with contributions. In this section we assume that the candidate policy platforms are exogenously determined. We consider a contribution game in which the contributors simultaneously choose which of two candidates to donate an indivisible unit of resource to. It should be noted that the characterized equilibria also exist in a continuous time model of sequential contributions in which contributors observe the

\footnotetext{
${ }^{5}$ The body of literature that deals with "network externalities" is broad. For a more detailed discussion of network effects and firm competition, see Economides (1996) and Katz and Shapiro (1994).
} 
history of previous contributions, and, in fact, the equilibria here are identical to the set of Markov-perfect equilibria in the dynamic game. ${ }^{6}$ Alternatively, it is clear that the current model is strategically identical to a sequential model of contributions in which contributors do not observe the history of contributions.

A continuum of contributors $I:=[0,1]$ are characterized by their ideal points $x(i) \in[0,1]$, while two candidates, $j \in\{0,1\}$, are characterized by their policy stances, $c_{j} \in[0,1]$. Without loss of generality, it is assumed that $c_{0}<c_{1}$. In modeling a general election between two candidates, one might justify the assumption of exogenous candidate locations by arguing that candidates are committed to the positions that they have staked out prior to the general election, in their respective primaries. Alternatively, one might argue that in "big money" elections, previous public service or campaigning serves as a constraint on candidates. In period 1 contributors $i \in I$ simultaneously contribute one resource unit to either candidate. Contributor $i$ 's choice is denoted by $b(i) \in\{0,1\}$. The aggregate contributions to candidate 1 is the Lebesgue integral $B_{1}=\int_{0}^{1} b(i) d i$, and since all voters must contribute, the contributions to candidate 0 are just $B_{0}=1-B_{1} .{ }^{7}$ The percentage of contributions to candidate 1 is denoted $R=B_{1} /\left(B_{1}+B_{0}\right)$. In period $t=2$ an election occurs. The winner of the election, $w \in\{0,1\}$, is randomly chosen and the random variable $w$ is assumed to have the conditional probabilities:

$$
\begin{aligned}
& \operatorname{prob}(w=1 \mid R)=p(R) \\
& \operatorname{prob}(w=0 \mid R)=1-p(R),
\end{aligned}
$$

where $p:[0,1] \rightarrow[0,1]$ is non-decreasing.

Contributors in this model care about the policy announcement of the candidate that they donate to, and whether they are donating to a winner. More specifically, we assume that contributor preferences are representable by utility functions of the form:

$$
u(x(i), b, w)=-\kappa\left|x(i)-c_{b}\right|+(1-\kappa) 1_{\{w=b\}} \quad \text { for } \kappa \in(0,1),
$$

and

$$
1_{\{w=b\}}= \begin{cases}1 & \text { if } w=b \\ 0 & \text { otherwise }\end{cases}
$$

Concerns might be raised over whether it is appropriate to assume that contributors care about the policy announcement of the candidate that they

\footnotetext{
${ }^{6}$ More precisely, the equilibria would be Markov perfect relative to the state space consisting of the current proportion of contributions going to each candidate.

${ }^{7}$ While it may seem unusual that we are not providing contributors with the option of withholding donations (and not contributing to any candidate), we seek to understand the behavior of those political activists who (perhaps due to their financial situations, ideological convictions, etc.) wish to participate in the process, and hence are very likely to give to one candidate over another, rather than none at all.
} 
donate to, rather than the actual policy that will be implemented following the election (which could, but will not necessarily, correspond to the policy stance of their favored candidate). It should be noted, however, that in a model with a large population of contributors where each makes small contributions (such as this one), if contributors care both about influencing the final policy and the position of the candidate they are supporting, as well as the desire to contribute to the winner, then the strategic choices will be influenced only by the latter two concerns as the probability that any one contribution decision will affect the outcome is 0 . On the other hand, if contributors care about the policy of the winning candidate and desire to contribute to the winner, but do not care about the policy of the candidate they support, then the strategic choices will be influenced only by the desire to contribute to the winner - again the action of an individual contributor cannot affect the outcome. Thus, the parameterization we consider captures the relevant strategic incentives of contributors that do care about the final policy, but are aware that their contributions alone will not affect the outcome. In large stakes elections with a high volume of contributions by individuals constrained to make small donations this assumption is reasonable.

Contributor $i$ 's ideal point, $x(i)$, is drawn from the strictly increasing probability distribution $F(\cdot)$ on support $[0,1]$, and all contributors' ideal points are assumed to be independent. Invoking a law of large numbers (Judd, 1985), we express the median contributor's ideal point by $m:=F^{-1}\left(\frac{1}{2}\right){ }^{8}$

The assumption that the winner is determined by a lottery with probabilities $p(R)$ and $1-p(R)$ is a reduced-form representation of voting. Since the function $p(R)$ is not specified, many different election games might fall into the guise of this model.

\subsection{Interpretations/Examples}

Given the above setup of the model, two interesting interpretations are the following:

Case 1. Suppose that the population of voters and the population of contributors are identical. Voters $I$ simultaneously vote after learning $R$ and the winner is determined by simple majority rule. Because the preferences $u(x, b, w)$ are well defined (which are similar to those in Morton and Myerson, 2000), voting strategies are well defined. Moreover, in subgames

\footnotetext{
${ }^{8}$ Judd shows that it is possible to construct probability spaces with a continuum of i.i.d. random variables for which a law of large numbers is well defined and holds. We embrace Judd's recommendation that suggests "that working economists assume that they have an extension of the Kolmogorov measure which satisfies the law of large numbers when they use these continuum models" (p. 24).
} 
following contributions, each contributor voting for the candidate they contributed to is a best response. In such a scenario, an appropriate assumption about $p(R)$ is the following:

$$
p(R)= \begin{cases}1 & \text { if } R \geq \frac{1}{2} \\ 0 & \text { otherwise. }\end{cases}
$$

The basic model with equation (4) is therefore a reduced-form representation of this contribution and voting game.

Case 2. Another possible interpretation (similar in spirit to Baron, 1994), is that there are actually two populations of voters, a continuum of wellinformed agents, that are endowed with resources, and a larger continuum of uninformed agents, that are somehow influenced exclusively by money. If the uninformed agents vote as a block probabilistically with law

$$
\begin{aligned}
& \operatorname{prob}(v=1 \mid R)=q(R) \\
& \operatorname{prob}(v=0 \mid R)=1-q(R),
\end{aligned}
$$

where $q:[0,1] \rightarrow[0,1]$ is non-decreasing, then the induced probabilities of victory will be consistent with the assumptions of the model being analyzed.

\section{ANALYSIS OF THE CONTRIBUTIONS GAME}

\subsection{Equilibrium Existence and Uniqueness}

A pure strategy Nash equilibrium to the contributions game involves each contributor simultaneously selecting the optimal candidate to support given a correct conjecture about the aggregate contribution behavior of the pool of contributors. For $\kappa<1$ contributors care about the odds that a candidate wins and thus for some contributors the optimal decision may actually hinge on the conjectured ratio. A measurable best-response mapping $b(x(i), R)$ : $[0,1]^{2} \rightarrow\{0,1\}$ can be characterized by Lebesgue-measurable contour sets, $b(1)^{-1}=\{x, R: b(x, R)=1\}$ and $b(0)^{-1}=\{x, R: b(x, R)=0\}$. Because contributors have a binary choice, $b(0)^{-1}=[0,1]^{2}-b(1)^{-1}$.

Definition 1. A Lebesgue-measurable subset $b(1)^{-1} \subset[0,1]^{2}$ represents a symmetric profile of best responses if:

$$
\begin{array}{ll}
E u(x, 1, w) \geq E u(x, 0, w) & \text { for all }(x, R) \in b(1)^{-1} \\
E u(x, 1, w) \leq E u(x, 0, w) & \text { for all }(x, R) \in[0,1]^{2}-b(1)^{-1} .
\end{array}
$$

Substituting the utility functions into this condition yields the following result.

Lemma 1. A Lebesgue-measurable subset $b(1)^{-1} \subset[0,1]^{2}$ represents a symmetric profile of best responses if: 


$$
\begin{aligned}
& -\kappa\left|x-c_{1}\right|+(1-\kappa) p(R) \geq-\kappa\left|x-c_{0}\right|+(1-\kappa)(1-p(R)) \\
& \quad \text { for all }(x, R) \in b(1)^{-1} \\
& -\kappa\left|x-c_{1}\right|+(1-\kappa) p(R) \leq-\kappa\left|x-c_{0}\right|+(1-\kappa)(1-p(R)) \\
& \quad \text { for all }(x, R) \in[0,1]^{2}-b(1)^{-1} .
\end{aligned}
$$

The next result establishes the existence of a symmetric profile of best responses, and demonstrates that they have connected contour sets. Specifically, the lemma characterizes a curve representing the ideal point and proportion pairs in $[0,1]^{2}$ that make a contributor indifferent between donating to candidate 0 or 1 .

Lemma 2. A symmetric profile of best responses exists, and the sets $b(1)^{-1}$ and $b(0)^{-1}$ are connected with the closure of $b(1)^{-1}$ equal to $\{(x, R) \in[0,1]: x \geq z(R)\}$ for

$$
z(R):= \begin{cases}0 & \text { if } \xi(R)<c_{0} \\ \xi(R) & \text { if } \xi(R) \in\left[c_{0}, c_{0}\right] . \\ 1 & \text { if } \xi(R)>c_{1}\end{cases}
$$

with

$$
\xi(R)=\left(\frac{1-\kappa}{\kappa}\right)\left(\frac{1}{2}-p(R)\right)+\frac{c_{0}+c_{1}}{2} .
$$

Figure 1 graphs a typical shape of the sets $b(1)^{-1}$ and $b(0)^{-1}$. Inspection indicates that for individuals with high ideal points, most levels of $R$ will

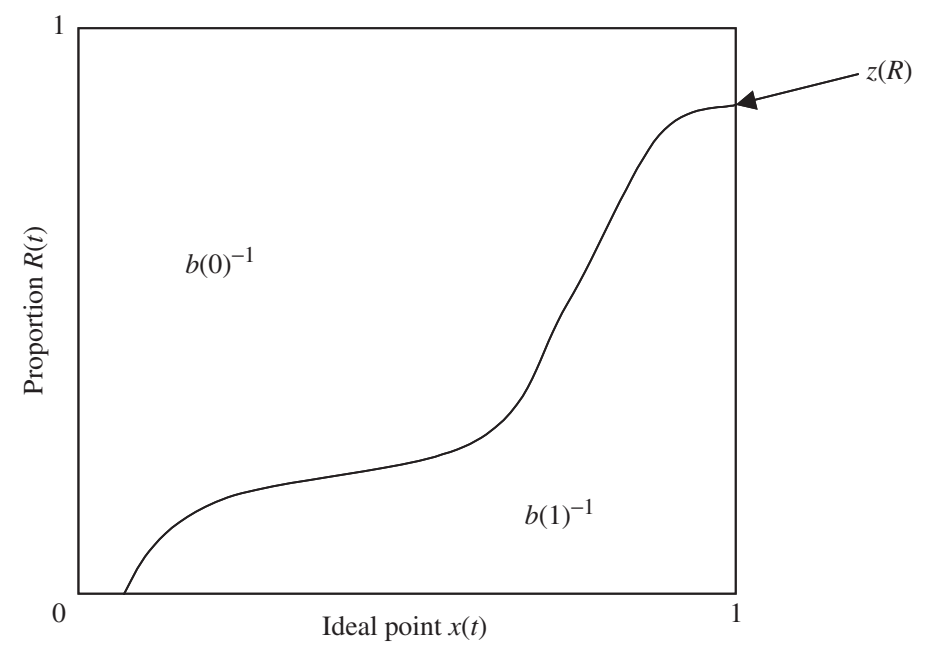

Figure 1. Payment schedules. 
induce a contribution to candidate 1 . Unsurprisingly when $p(R)=\frac{1}{2}$ or $\kappa$ is close to 1 contributors with ideal points to the right of the cutpoint $\left(c_{0}+c_{1}\right) /$ 2 give to candidate 1 . Alternatively, when $\kappa$ is sufficiently small, all contributors give to 1 if $p(R) \geq \frac{1}{2}$.

The fact that the contour sets are connected is important because it implies that for a given $R$, the sets $b(1)^{-1}$ and $b(0)^{-1}$ are separate intervals. Hence the probability that a randomly selected contributor will contribute to candidate 1 if she anticipates that the contribution proportion will be $R$ can be expressed simply as $(1-F(z(R))$ ). Invoking a law of large numbers (see footnote 8 ), this expression is also the proportion of contributors that give to candidate 1 (if agents optimize given the conjecture that $R$ is the proportion of agents that contribute to candidate 1 ).

Definition 2. A symmetric pure strategy Nash equilibrium (NE) is a symmetric profile of best responses, $b(1)^{-1} \subset[0,1]^{2}$, and a proportion, $R^{*} \in[0,1]$, subject to

$$
R^{*}=1-F\left(z\left(R^{*}\right)\right)
$$

Having established the above lemmas, establishing the existence of an NE is a straightforward application of Tarsky's fixed-point theorem. ${ }^{9}$

Proposition 1. In the contribution game, an NE exists.

We now address the question of equilibrium uniqueness. While it is clear that the function $z(\cdot)$ is unique, the optimal action when a contributor is indifferent between the two candidates is not uniquely specified. Since the measure of contributors that are indifferent is 0 , this issue is ignored, and attention is focused on the more interesting issue - the fixed points $R^{*}$ solving (9) may not be unique. In the case that $R^{*}$ is not unique, there are several beliefs that are self-fulfilling under the same best responses. It should be noted that in the case of multiple fixed points, the behavior of the system is not completely determined by anything intrinsic to the modeled political environment (e.g. candidate locations, money). As the following examples demonstrate, different parameterizations can have starkly different uniqueness properties:

An Example with a Continuum of Equilibria. Let $p(R)=R, F(x)=x, c_{1}=1$, $c_{0}=0, \kappa=\frac{1}{2}$. In this perfectly symmetric case, equation (9) is

$$
R=1-z(R)
$$

\footnotetext{
${ }^{9}$ We refer to a Nash equilibrium as being "symmetric" in that we are focusing on equilibria in which agents of the same type employ the same strategy.
} 
This reduces to the condition

$$
R=R,
$$

so that all $R^{*} \in[0,1]$ are supportable in equilibrium, meaning that all contribution combinations are supportable, despite the fact that the candidates are ideological opposites of each other.

An Example with Three Equilibria. Let $p(R)=R$ and $F(x)=x$ with $c_{1}=\frac{7}{8}, c_{0}=\frac{1}{8}, \kappa=\frac{1}{6}$. Under these assumptions, the equilibrium condition reduces to:

$$
R= \begin{cases}0 & \text { if } R<\frac{17}{40} \\ 1-5\left(\frac{1}{2}-R\right)-\frac{1}{2} & \text { if } R \in\left[\frac{17}{40}, \frac{23}{40}\right] \\ 1 & \text { if } R>\frac{23}{40}\end{cases}
$$

Accordingly, the values $R=0$ and $R=1$ are solutions. In addition the interior root $R=\frac{1}{2}$ solves

$$
R=1-5\left(\frac{1}{2}-R\right)-\frac{1}{2} .
$$

So that the solutions are $R^{*} \in\left\{0, \frac{1}{2}, 1\right\}$.

In general, neither of these phenomena can be ruled out; but there is, however, a distinction between the case of a continuum of equilibria and a finite number of equilibria. The former equilibria follow from knife-edge cases of parametric specifications, whereas the latter follow from more generic assumptions about the relevant functions $p(R), F(x)$, and the parameters $c_{1}, c_{0}$, and $\kappa$. This point is demonstrated in the next subsection.

In the above examples 0 and 1 are equilibrium proportions. A straightforward sufficient condition for extremal equilibria of this form exists. Given the monotonicity of $1-F(z(\cdot))$ in $R$, it is sufficient to analyze $1-F(z(1))$ and $1-F(z(0))$. Given the strict monotonicity of $F(\cdot)$, in order for $R^{*}=1$ to occur in equilibrium it must be the case that $\xi(1) \leq 0$; and in order for $R^{*}=0$ to occur in equilibrium it must be the case that $\xi(0) \geq 1$. This yields the result:

Proposition 2. (i) $R^{*}=1$ is an equilibrium if and only if

$$
p(1) \geq \frac{\kappa}{1-\kappa}\left(\frac{c_{1}-c_{0}}{2}\right)+\frac{1}{2} .
$$

(ii) $R^{*}=0$ is an equilibrium proportion if and only if

$$
p(0) \leq \frac{\kappa}{1-\kappa}\left(\frac{c_{0}-c_{1}}{2}\right)+\frac{1}{2} .
$$

Taking the case of $p(0)=0$ and $p(1)=1$ yields the following sufficient condition to ensure that no boundary equilibria exist: 
Proposition 3. (i) If

$$
\frac{\kappa}{1-\kappa}\left(\frac{c_{0}-c_{1}}{2}\right)+\frac{1}{2}<0 \quad \text { and } \quad 1<\frac{\kappa}{1-\kappa}\left(\frac{c_{1}-c_{0}}{2}\right)+\frac{1}{2}
$$

then every equilibrium proportion, $R^{*}$, is in $(0,1)$.

Informally, if it is the case that getting all of the campaign contributions ensures victory, and contributors value donating to the winner sufficiently high, then there are equilibria in which all of the resources go to either candidate. Conversely, when the importance of donating to the winner is less extreme, and resources are less decisive in terms of election prospects, there will only be equilibria with resources going to both candidates.

\subsection{Comparative Statics (Smooth Analysis)}

In this section the assumptions of the basic model are modified to facilitate the use of the calculus to conduct comparative static analysis in order to characterize how equilibrium contribution proportions vary with changes in the candidates' ideal points, contributors' preferences, etc. Specifically, the smooth version of the model has $F(\cdot)$ and $p(\cdot)$ as continuous, differentiable, and strictly increasing functions. No other modifications are made, but the existing notation is slightly augmented in order to facilitate exposition. More specifically, the vector $\theta=\left(\kappa, c_{0}, c_{1}\right)$ is meant to denote a particular parameterization of the model, wherein $\theta_{1}=\kappa, \theta_{2}=c_{0}$, and $\theta_{3}=c_{1}$. The notation $z(R ; \theta)$ and $\xi(R ; \theta)$ is used in place of $z(R)$ and $\xi(R)$.

Definition 3. Let $\mathscr{R}^{*}(\theta)$ denote the set of solutions to equation (9).

Proposition 4. Fix the smooth functions $p(R)$ and $F(x)$; then for all but a set of parameters $\left(c_{0}, c_{1}, \kappa\right) \in[0,1]^{3}$ that have Lebesgue measure 0 , the number of equilibria is finite and each supportable $R^{*}$ is locally unique.

The intuition behind this result may be attained graphically by considering Figure $2 .{ }^{10} \mathrm{~A}$ non-transversal crossing of $(1-F(z(R ; \theta)))$ and the $45^{\circ}$ line is exhibited. But a small perturbation of the curve $(1-F(z(R ; \theta)))$ to $\left(1-F\left(z\left(R ; \theta^{\prime}\right)\right)\right)$ causes all non-transversal intersections to disappear. Hence, in virtually all cases there is a finite set of locally unique contribution proportions that are likely to occur.

In the following analysis a generic subset of the parameter space is considered as only locally unique (or isolated) equilibria are analyzed.

\footnotetext{
${ }^{10}$ Since the functions $p(R)$ and $F(x)$ are parameters of the model, a stronger result is desirable: that in the product space of possible functions $(p(R), F(x))$, and parameters $\left(c_{0}, c_{1}, \kappa\right)$, on a generic subset of the parameter space the fixed points $R^{*}$ are locally unique. This analysis is not included as it offers no additional insight.
} 


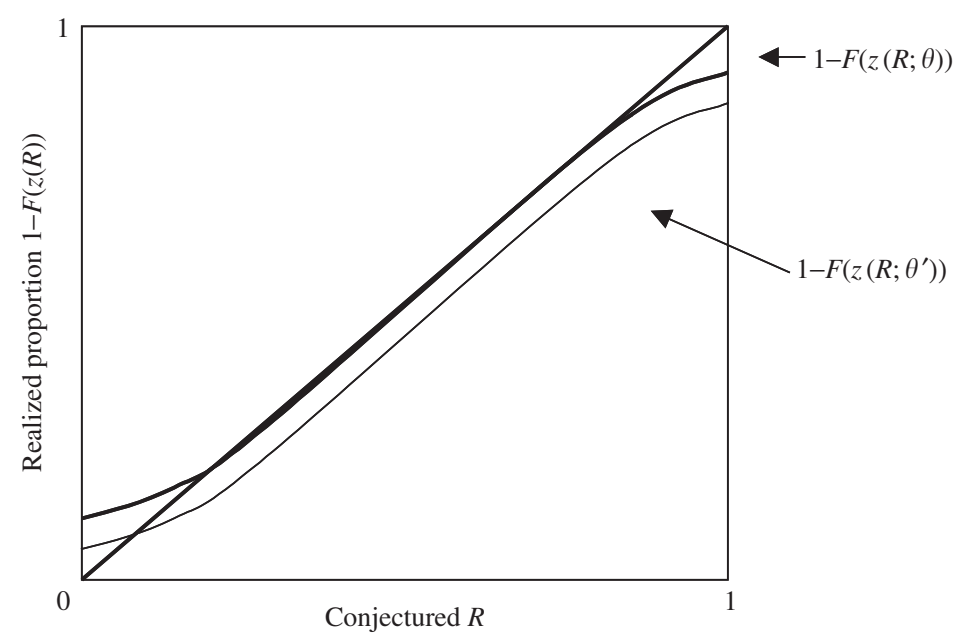

Figure 2. Continuum of equilibria.

Inspection of (8) indicates that while $z(R ; \theta)$ is not everywhere continuous (let alone differentiable) in $R$ or $\theta$ the points of discontinuity can be easily treated. Note that the discontinuities occur when $1-F(z(R ; \theta))$ jumps from 0 to $1-F\left(c_{0}\right)$, or from $1-F\left(c_{1}\right)$ to 1 . Accordingly, the only possible fixed points for which smooth analysis cannot be used are $\left\{0,1-F\left(c_{0}\right), 1-F\left(c_{1}\right), 1\right\}$. In this section we focus on interior fixed points that result from transversal crossings and use the implicit function theorem to attain comparative statics. We call such fixed points interior generic fixed points. Consideration of Figure 3 indicates that when the graph of $(1-F(z(R ; \theta)))$ shifts upwards, the extent to which $R^{*}$ increases depends on whether the graph of the curve $(1-F(z(R ; \theta)))$ crosses the $45^{\circ}$ line from above or below. In the event that $(1-F(z(R ; \theta)))$ crosses the $45^{\circ}$ from above,

$$
-\frac{\partial F(z(R ; \theta))}{\partial R}<1
$$

whereas

$$
-\frac{\partial F(z(R ; \theta))}{\partial R}>1
$$

for the case where it crosses the $45^{\circ}$ line from below.

Definition 4. Let upper crossing fixed points be defined as

$$
U(\theta):=\left\{x \in \mathscr{R}^{*}(\theta): F^{\prime}(z(R))\left(\frac{1-\kappa}{\kappa}\right) p^{\prime}(R)<1\right\}
$$




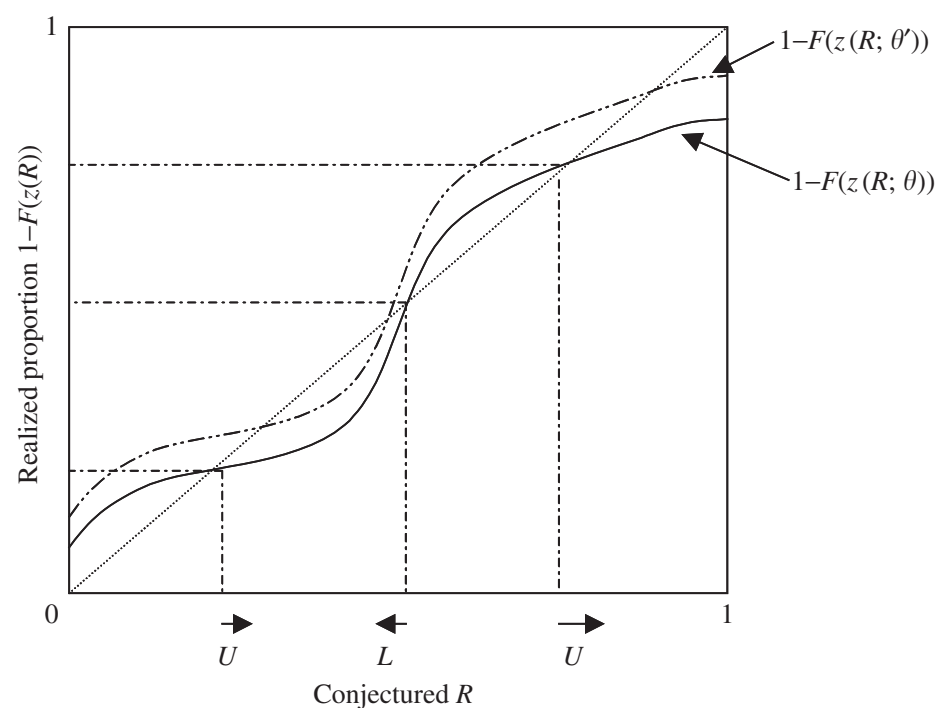

Figure 3. Comparative statics.

and let lower crossing fixed points be defined as

$$
L(\theta):=\left\{x \in \mathscr{R}^{*}(\theta): F^{\prime}(z(R))\left(\frac{1-\kappa}{\kappa}\right) p^{\prime}(R)>1\right\} .
$$

If all fixed points are interior it is obvious that an odd number of fixed points exist and $U(\theta)$ is generically non-empty. Moreover, in these cases the minimal and maximal elements of $\mathscr{R}^{*}(\theta)$ are in $U(\theta)$. These fixed points are also more stable than those in $L(\theta)$ in the sense that they are absorbing. In other words, small deviations up from a stable fixed point proportion $R^{*}$, will result in equilibrium levels of support $\left(1-F\left(z\left(R^{*}\right)\right)\right)$ that are between the erroneously conjectured level and the equilibrium level. ${ }^{11}$ Conversely, for the non-stable levels, small errors in beliefs can cause the system to move far away from the equilibrium level. However, if boundary fixed points exist then it is possible for there to be either an even or an odd number of fixed points. This is true because the continuous and non-constant part of $z(R ; \theta)$ may start and end above or below the $45^{\circ}$ line. The next lemma demonstrates that to determine the sign of the comparative static $\partial R^{*} / \partial \theta_{i}\left(\theta_{i} \in\left\{\kappa, c_{0}, c_{1}\right\}\right)$ at a particular $R^{*}$, it is sufficient to find the sign of the derivative $\partial F(z(R ; \theta)) /$ $\partial \theta_{i}$ and determine whether $R^{*} \in U(\theta)$ or $L(\theta)$.

\footnotetext{
${ }^{11}$ Our employment of the term "stability" is consistent with the concept of asymptotic stability as discussed in Fudenberg and Tirole (1991, p. 25) and analyzed in various Cournot models by scholars such as Dixit (1986), Fisher (1961), and Hahn (1962).
} 
Lemma 3. In the smooth model at an interior generic fixed point $R^{*} \in \mathscr{R}^{*}(\theta)$, the comparative static $\partial R^{*} / \partial \theta_{i}$ exists and (i) if $R^{*} \in U(\theta)$ it has the opposite sign as the derivative $\partial F(z(R ; \theta)) / \partial \theta_{i}$, and (ii) if $R^{*} \in L(\theta)$ it has the same sign as the derivative $\partial F(z(R ; \theta)) / \partial \theta_{i}$.

While not interesting in its own right this result conforms to our graphical intuition, and will be useful in establishing subsequent results.

For simplicity, the next results are only stated for the fixed points in $U(\theta)$. The counterpart comparative statics for the non-stable $L(\theta)$ fixed points have the opposite sign. The first result examines how the equilibrium ratio of contributions changes as contributors' preferences become more policy oriented. Formally, this comparative static is the partial derivative $\partial R^{*} / \partial \kappa$.

Proposition 5. In the smooth model at an interior generic fixed point $R^{*} \in U(\theta), \partial R^{*} / \partial \kappa<(>) 0$ if $p\left(R^{*}\right)>(<) \frac{1}{2}$.

While this result is not immediately obvious, it does conform with intuition. Consider the highest contribution proportion, $R^{*}$; this fixed point is in $U(\theta)$ and if it satisfies $p\left(R^{*}\right)>\frac{1}{2}$ (so that candidate 1 is the projected winner), then the result states that if contributors' policy preferences increase in relative importance more support will go to the candidate that is projected to lose. This result is reasonable, as, when $R^{*}$ is high, there are contributors giving to candidate 1 despite her policy stance. As policy importance increases, however, some contributors substitute toward the candidate whose policy stance they prefer, regardless of her chances for victory. These findings are unambiguous, and demonstrate that equilibrium behavior changes in response to changes in policy importance depending on which candidate is the likely victor.

The following results explain how changes in the two candidates' policy stances, $c_{0}, c_{1}$ affect the equilibrium contribution proportion.

Proposition 6. In the smooth model at an interior generic fixed point $R^{*} \in U(\theta), \partial R^{*} / \partial c_{j}<0(j \in\{0,1\})$.

Hence, holding the weight on policy preferences constant, as either candidate moves further to the right, the proportion of contributions to candidate 1 decreases. Since candidate 0 is to the left of 1 this means that as either candidate moves towards the other, the proportion of contributions she receives increases. This makes sense, intuitively, given that as candidate 1 moves farther to the right (farther from the median contributor) he alienates potential donors. Conversely, as candidate 0 moves farther to the right he retains the support of those who were already contributing to him, while garnering additional support from some of the contributors located in between him and candidate 1 . 
Finally, we now consider the comparative statics of non-interior fixed points. From Proposition 2 we know that the boundary fixed points result when

$$
p(1) \geq \frac{\kappa}{1-\kappa}\left(\frac{c_{1}-c_{0}}{2}\right)+\frac{1}{2} \quad \text { or } \quad p(0) \leq \frac{\kappa}{1-\kappa}\left(\frac{c_{0}-c_{1}}{2}\right)+\frac{1}{2} .
$$

If the inequality is strict then it is easy to see that the fixed points are nonresponsive to local changes in the parameters $\theta$. Since for generic parameters neither inequality will hold with equality, we are left with the following result:

Corollary 1. Consider the set of games in which degenerate fixed points exist. On a generic subset, the degenerate fixed points are non-responsive to small enough changes in $\theta$.

\subsection{Contribution Analysis when $c_{0}=c_{1}$}

Having discussed equilibrium results for any generic $c_{0}, c_{1}$, we now consider what occurs in the contribution game for the specific case when $c_{0}=c_{1}$. Given that the canonical spatial location game (e.g. Downs, 1957) predicts that candidates will locate at the median voter's (in this case, median contributor's) ideal point and tie in the election, we seek to understand how robust this prediction is once we include money in the election. It is not difficult to see that in this case $\left\{(R, x) \in[0,1]^{2}: R>\frac{1}{2}\right\} \subset b^{-1}(1)$ and $\left\{(R, x) \in[0,1]^{2}: R<\frac{1}{2}\right\} \subset b^{-1}(0)$. We assume that when both $c_{0}=c_{1}$ and $p(R)=\frac{1}{2}$ under the conjectured $R$, each candidate gets an equal share of the contributions. This can be attained by either assuming that a law of large numbers holds, or allowing contributors to use non-symmetric strategies in this case of global indifference.

When candidates locate at the same position, the behavior of the model depends largely on whether one candidate is a priori favored over the other.

Definition 5. An electoral environment is neutral if $p\left(\frac{1}{2}\right)=\frac{1}{2}$, and it (a priori) favors $c_{0}\left(c_{1}\right)$ if $p\left(\frac{1}{2}\right)<(>) \frac{1}{2}$.

Informally, one might interpret a favorable electoral environment to be capturing the existence of an incumbency advantage. In most elections incumbents, ceteris paribus, are the a priori favored candidates.

Proposition 7. If $c_{0}=c_{1}$ then (1) an NE exists and 1 or 0 (or both) are contained in $\mathscr{R}^{*}$; (2) if the electoral environment is neutral then $\left\{0, \frac{1}{2}, 1\right\}=\mathscr{R}^{*}$; and (3) if $c_{0}\left(c_{1}\right)$ is (a priori) favored then any interior $R^{*}$ satisfies $R^{*}>(<) \frac{1}{2}$. 
Accordingly, there is always an equilibrium where one of the two candidates gets all of the contributions. With neutral environments, contributions are either maximally lopsided or maximally symmetric. Part (3) of the proposition is somewhat surprising. It states that in a non-neutral environment in which both candidates get a non-trivial portion of the contributions, the favored candidate will get less than half of the contributions. The intuition behind the result is straightforward. When candidates locate at the same position and both candidates receive contributions, all contributors must be indifferent between donating to either candidate. In order for contributors to be indifferent between the two candidates they must believe that the candidates have equal chances of winning the election. Accordingly, the a priori favored candidate must be getting less than half of the donations.

At first glance, this result differs from Morton and Myerson who, in viewing incumbency as a coordination device for voters, argue that all contributions should flow to an incumbent candidate. The difference arises because Morton and Myerson implicitly assume that the environment is neutral, and argue that the focal equilibrium is the one in which the incumbent gets all of the support. We treat incumbency not as a mechanism to select focal equilibria, but as a factor that influences the relationship between contributions and voting (i.e. the incumbent is a priori favored). ${ }^{12}$ That being said, our, and Morton and Meyerson's, model highlight a finding that is seemingly robust to different assumptions about the preferences of voters and/or contributors: the candidate that is viewed (by various criteria) as favored by the voters may receive fewer contributions in races where the two candidates stake out (nearly) identical locations.

If we believe that incumbents are a priori favored candidates then this result has a direct implication for the relationship between incumbents' war chests and close races. In races that are electorally and spatially close (i.e. races where candidates locate near each other and the race is not a landslide), the incumbent candidate should receive fewer contributions than the challenger.

\section{THE CANDIDATE LOCATION GAME}

Whereas the previous analysis focused on equilibrium contribution proportions following from exogenous candidate locations, this section takes a step backwards by augmenting the basic model to include a candidate platform selection stage prior to the contribution game. If we believe that candidates are not committed to the positions staked out during the primaries (or, alternatively, they are unconstrained in their choices during the primaries), this model provides greater insight into the relationship between campaign contributions, candidate locations, and electoral outcomes. The

\footnotetext{
${ }^{12}$ It should be noted that under the assumption incorporated in part (3), the only robust equilibria involve all contributions going to one candidate.
} 
game begins with simultaneous selection of policy platforms $c_{j} \in[0,1]$ for $j \in\{0,1\}$. Then the basic game of the previous section is played. Candidates are assumed to be office seekers whose preferences can be represented by the following utility functions:

$$
\begin{aligned}
& u_{1}\left(c_{0}, c_{1}\right)=p\left(R^{s}\left(c_{0}, c_{1}\right)\right) \\
& u_{0}\left(c_{0}, c_{1}\right)=1-p\left(R^{s}\left(c_{0}, c_{1}\right)\right) .
\end{aligned}
$$

The notation $R^{S}\left(c_{0}, c_{1}\right)$ is used to denote a particular selection from the set $\mathscr{R}^{*}\left(\kappa, c_{0}, c_{1}\right)$ of equilibrium contribution proportions resulting from the stances $\left(c_{0}, c_{1}\right)$.

Since the set $\mathscr{R}^{*}\left(\kappa, c_{0}, c_{1}\right)$ is not generally a singleton, pinning down behavior of the candidates with an equilibrium concept involving optimization of expected utility requires determining how candidates form beliefs about which $R^{*}$ will be realized following a particular profile $\left(c_{0}, c_{1}\right)$. Clearly, the support of these beliefs should be $\mathscr{R}^{*}\left(\kappa, c_{0}, c_{1}\right)$. For the remainder of this section $\kappa$ will be suppressed and the following assumptions will be employed when appropriate:

$$
\begin{aligned}
& p(0)<\frac{1}{2}<p(1) \\
& p(0)<\frac{\kappa m}{1-\kappa}+\frac{1}{2} \\
& p(1)>\frac{\kappa m}{1-\kappa}+\frac{1}{2},
\end{aligned}
$$

where $m$, as defined earlier, is the median contributor's ideal point. Inequality (A1) is interpreted as ensuring that a candidate is more likely to lose than win when he receives no funding. Given Proposition 2 this inequality also implies that when candidates are nearly identically located there are equilibria to the contribution game in which all of the resources go to either candidate. Inequalities (A2) and (A3) state that when the candidates are nearly equally spaced from the median contributor there are equilibria to the contribution game in which all of the resources go to either candidate.

In determining which assumptions to employ about candidate beliefs, one defensible approach is to assume that both candidates form the same beliefs about the lottery over $\mathscr{R}^{*}\left(c_{0}, c_{1}\right)$. In this case candidate beliefs are characterized by a conditional (belief) distribution function $G\left(\cdot ; c_{0}, c_{1}\right)$ that is constrained to have support $\mathscr{R}^{*}\left(c_{0}, c_{1}\right)$. Hence, $G\left(\cdot ; c_{0}, c_{1}\right)$ is a mapping, $G\left(\cdot ; c_{0}, c_{1}\right):[0,1]^{2} \rightarrow\left\{\right.$ distributions on $\left.\mathscr{R}^{*}\left(c_{0}, c_{1}\right)\right\}$.

Definition 6. A common candidate platform equilibrium (CCPE) is a belief mapping and a pair $\left(c_{0}^{*}, c_{1}^{*}\right)$ for which: 


$$
\begin{aligned}
& c_{1}^{*} \in \arg \max _{c \in[0,1]}\left\{\int P(R) d G\left(R ; c_{0}^{*}, c\right)\right\} \\
& c_{0}^{*} \in \arg \max _{c \in[0,1]}\left\{1-\int P(R) d G\left(R ; c, c_{1}^{*}\right)\right\} .
\end{aligned}
$$

An example involving one pool of individuals that first makes contribution decisions and then votes after the candidates chose their platforms will clarify this concept.

Corollary 2. Assume that contributor ideal points are uniformly distributed on the policy space, $F(x)=x$, that

$$
P(R)= \begin{cases}1 & \text { if } R>\frac{1}{2} \\ \frac{1}{2} & \text { if } R=\frac{1}{2} \\ 0 & \text { if } R<\frac{1}{2}\end{cases}
$$

and $\kappa \in(0,1)$. This example is the reduced-form representation of a game in which following the contribution stage all contributors simultaneously vote sincerely and the winning candidate is determined by majority rule. In this example it is not difficult to verify that

$$
\mathscr{R}^{*}\left(c_{0}, c_{1}\right)= \begin{cases}\{1\} & \text { if }\left|c_{0}-\frac{1}{2}\right|>\left|c_{1}-\frac{1}{2}\right| \\ \left\{0, \frac{1}{2}, 1\right\} & \text { if }\left|c_{0}-\frac{1}{2}\right|=\left|c_{1}-\frac{1}{2}\right| \\ \{0\} & \text { otherwise. }\end{cases}
$$

So one degenerate candidate belief is

$$
G\left(R ; c_{0}, c_{1}\right)= \begin{cases}1 & \text { if } R=1 \text { and }\left|c_{0}-\frac{1}{2}\right|>\left|c_{1}-\frac{1}{2}\right| \\ 1 & \text { if } R=\frac{1}{2} \text { and }\left|c_{0}-\frac{1}{2}\right|=\left|c_{1}-\frac{1}{2}\right| \\ 1 & \text { if } R=0 \text { and }\left|c_{0}-\frac{1}{2}\right|<\left|c_{1}-\frac{1}{2}\right| \\ 0 & \text { otherwise. }\end{cases}
$$

Under this candidate belief, the policy stances $c_{0}=c_{1}=\frac{1}{2}$ are supportable as an equilibrium. To see that no unilateral deviation from these stances is desirable, note that if candidate 0 unilaterally deviates she will lose the election with probability 1 , and if candidate 1 unilaterally deviates she will win the election with probability 0 . In fact, in this example the profiles $\left(\frac{1}{2}, \frac{1}{2}\right)$ are simultaneous best responses for any belief mapping.

Since Von Neumann-Morgenstern utility functions are only defined up to affine transformations, for a fixed-belief mapping the candidate platform selection game (with common candidate beliefs) is equivalent to a zero-sum game. Accordingly the following convenient result applies. 
Lemma 4. Fix the common belief mapping $G\left(\cdot ; c_{0}, c_{1}\right)$. If $\left(c_{0}^{*}, c_{1}^{*}\right)$ and $G\left(\cdot ; c_{0}, c_{1}\right)$ are a CCPE with equilibrium payoffs of $(p)$ and $(1-p)$ then in every CCPE with the same belief mapping the payoffs are also $(p)$ and $(1-p)$.

The lemma states that modulo candidate beliefs, the equilibrium payoffs of the game are unique. The existence of CCPE is now considered. The first result establishes that under suitable conditions there is a CCPE with a median contributor outcome: ${ }^{13} c_{0}=c_{1}=F^{-1}\left(\frac{1}{2}\right):=m$.

Proposition 8. If (A1)-(A3) hold and $F(\cdot)$ and $p(\cdot)$ are smooth then there is a CCPE in which $c_{0}=c_{1}=m$.

Note that the result states that there is a common belief mapping in which median policies are equilibrium candidate platforms (in the sense of CCPE). This does not generally hold for common belief mappings. In fact, it is not the case that every common belief mapping can be supported in a CCPE. One problem is that for an arbitrary $G\left(\cdot ; c_{0}, c_{1}\right)$ the induced payoff $\int P(R) d G\left(R ; c_{0}, c_{1}\right)$ need not be continuous in the choice variables. Even if a common belief mapping induces continuity, it may not be the case that the solutions to (15) are single-valued or convex valued. ${ }^{14}$

It should be noted that Lemma 4 cannot be applied to establish that for every common belief mapping that supports a CCPE, the equilibrium probability that candidate 1 wins is $\frac{1}{2}$. This does not hold because Lemma 4 only states that for a fixed common belief mapping every stable platform profile yields the same payoffs. When we are free to choose belief mappings in the construction of CCPE, we cannot be assured that all CCPE are payoff equivalent. We can show that under the somewhat natural belief mapping used in the previous construction all CCPE involve $c_{0}=c_{1}=m$.

Proposition 9. Assume (A1). Any CCPE with the common belief mapping defined as

$$
G\left(R ; c_{0}, c_{1}\right)= \begin{cases}1 & \text { if } R=p^{-1}\left(\frac{1}{2}\right) \text { and } c_{0}=c_{1} \\ 1 & \text { if } R=\inf \left\{\mathscr{R}^{*}\left(c_{0}, c_{1}\right)\right\} \text { and } 0<\left|c_{0}-m\right| \leq\left|c_{1}-m\right| \\ 1 & \text { if } R=\sup \left\{\mathscr{R}^{*}\left(c_{0}, c_{1}\right)\right\} \text { and }\left|c_{0}-m\right|>\left|c_{1}-m\right| \\ 0 & \text { otherwise }\end{cases}
$$

involves $c_{0}=c_{1}=m$.

Having established the existence of an equilibrium with $c_{0}=c_{1}=m$, a further question to consider is whether all CCPE have $c_{0}=c_{1}=m$ when (A1)-(A3)

\footnotetext{
${ }^{13}$ This result is analogous to the conventional median voter result.

${ }^{14} \mathrm{As}$ such, the standard proof technique of utilizing the Theorem of the Maximum and Kakutani's fixed point theorem may fail.
} 
hold. It turns out that as long as policy is not too important to contributors, then any profile of candidate stances is supportable in a particularly simple equilibrium.

Proposition 10. If $p(0)=0, p(1)=1$, and $\kappa \leq \frac{1}{2}$, then any profile $\left(c_{0}, c_{1}\right) \in[0,1]^{2}$ is supportable in a CCPE.

A few comments about the proof (in the Appendix) are in order. It is important to note that the function $\psi\left(c_{0}, c_{1}\right)$, (defined in the proof) which denotes the probability that 1 wins when the profile is $\left(c_{0}, c_{1}\right)$ need not be constant to support the above construction. In fact a strict equilibrium requires only that $\psi\left(c_{0}, c_{1}\right)$ jointly attain a unique minimum on the set $\left\{(x, y) \in[0,1]^{2}: y=c_{1}^{\prime}\right\}$ and a unique maximum on the set $\left\{(x, y) \in[0,1]^{2}\right.$ : $\left.x=c_{0}^{\prime}\right\}$ at $\left(c_{0}^{\prime}, c_{1}^{\prime}\right)$. Under such a mapping, either candidate associates a unilateral move with a decrease in the probability of victory. Figure 4 depicts such a function.

The equilibria discussed above are undesirable in the sense that they hinge on candidates believing only that degenerate contribution levels will occur. We may ask how indeterminate the election is if we require that contribution levels be in the interior of the unit interval. In general, requiring only interior proportions to be in the support of candidate beliefs does not pin down the behavior of the model very much. The reason is that as long as $\mathscr{R}^{*}\left(c_{0}, c_{1}\right)$ is not a singleton for a large set of profiles $\left(c_{0}, c_{1}\right)$, the construction used in the proof of Proposition 10 can be replicated to support many equilibria.

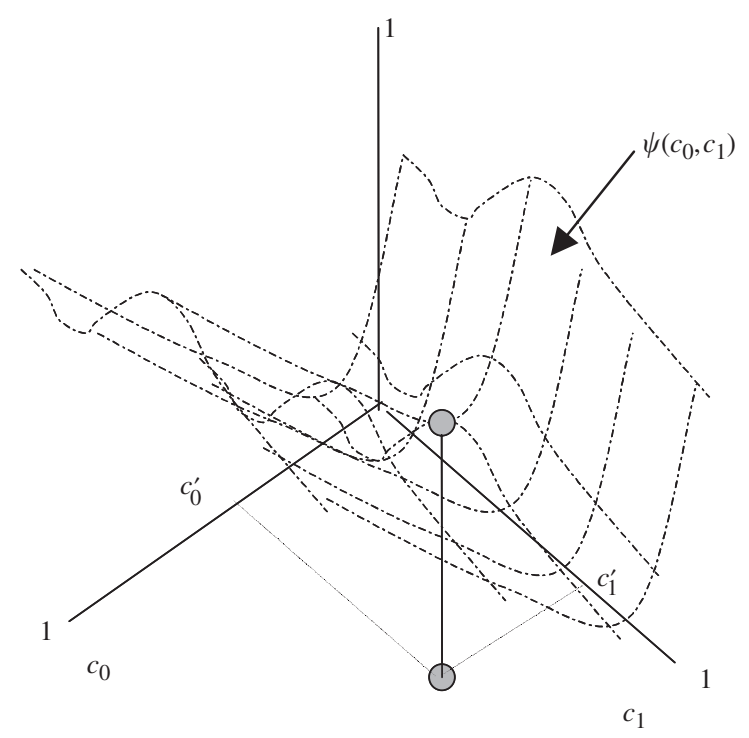

Figure 4. Supporting beliefs. 
In contrast to Morton and Myerson's finding that in the complete information model robust equilibria involve policy convergence and one candidate receiving all of the contributions, our results indicate that there is no natural reason to select equilibria involving candidate platforms that correspond to the median voter's ideal point. From an empirical point of view our result is encouraging, as many elections involve non-identical candidate platforms (Ansolabehere et al., 2001), and it is rarely the case that two candidate contests involve the exorbitant resource difference predicted by the extreme equilibria in which one candidate gets all of the contributions (Jacobson, 1992). However, the current model also has a substantial disadvantage. It offers many predictions, making the task of testing difficult. As such, it is necessary to test more subtle implications of the model to assess its empirical validity. This point will be discussed in the conclusion.

\subsection{An Extension: Voters Who Care About Policy and Contributions}

It may seem troublesome that the candidates' re-election functions, as modeled in this section, do not hinge explicitly on their policy location. While it is true that the models analyzed include cases that can sneak policy in the re-election function (as noted in earlier examples), the results above do not hold generally when we consider a more policy-motivated electorate. That being said, if we define a candidate's re-election function as $p\left(R, c_{0}, c_{1}\right)$ and assume that some money is needed to win the election then the results in this section can be immediately extended:

Corollary 3. If $\kappa \leq \frac{1}{2}$ and the following conditions are satisfied,

$$
\begin{aligned}
& p\left(0, c_{0}, c_{1}\right)=0 \text { for all }\left(c_{0}, c_{1}\right) \\
& p\left(1, c_{0}, c_{1}\right)=1 \quad \text { for all }\left(c_{0}, c_{1}\right),
\end{aligned}
$$

then any profile $\left(c_{0}, c_{1}\right) \in[0,1]^{2}$ is supportable in a CCPE.

Intuitively, (A4) and (A5) can be interpreted to mean that regardless of what locations the candidates choose, if either candidate receives all available contributions, he will win. This seems like a reasonable assumption, and serves to demonstrate that the framework of the existing model can be easily altered to accommodate the policy preferences of voters. We do not include the proof because it is easy to see that the function $\psi\left(c_{0}, c_{1}\right)$ used in the proof of Proposition 10 can be used to attain the corollary.

\section{CONCLUSION}

We began this paper by asking what role money could play in electoral competition when contributors care both about policy and the viability of a possible candidate. By analyzing a contribution game with network 
externalities, we have established necessary and sufficient conditions for the existence of equilibria in which unpopular candidates (on policy grounds) may be electorally viable. These findings suggest that the presence of contributions in electoral competition may cause a disconnect between the policy preferences of the electorate and the choices that they make. For the case of endogenous candidate locations the story is a bit more complex, but the approach taken here is a step in the right direction. We have demonstrated that an equilibrium exists where both candidates locate at the median contributor's ideal point. However, median policies are far from the unique, clear prediction of the model. The existence of money and preferences of the form considered introduces an indeterminacy to the model - nearly anything can be supported. Of course, future work at refining the candidate beliefs may lead to narrower predictions.

One interpretation of this study, then, is that money and preferences that exhibit network externalities open up the door for media and personality effects, even if voters/contributors are not attentive to the media or do not greatly value candidate personality. In other words, these devices may simply resolve the problem of choosing an equilibrium. In such a world, the candidate beliefs may be interpreted as focusing on how the media will respond to various types of elections, and therefore which equilibrium will be chosen. The fact that this finding is present in environments where voter and contributor preferences over policy are well behaved and admit a Condorcet winner, is suggestive that contributions may be inefficient. This finding is in contrast to the conclusion of Morton and Myerson, indicating that the assumptions about contributor and voter preferences (upon which the two models differ) are very important.

In the reasonable equilibria where both candidates locate at the median contributor's ideal point, a counterintuitive finding emerges: the a priori favored candidate will receive fewer contributions when the election is very close. While this finding does not comport with conventional wisdom, it seems to fit nicely with recent electoral dynamics in the 2000 presidential race. As discussed in the introduction, Gore, the expected favorite, appeared to be very similar to Bush on many policy dimensions while at the same time receiving fewer contributions than Bush. While this case is illustrative in supporting the theory, recently available data sources might be employed to conduct large-sample empirical analysis to deduce whether this theoretically postulated claim generally holds in close races. The National Political Awareness Test (NPAT) ${ }^{15}$ data offer an ideal opportunity for developing ideological positions for both incumbents and challengers on a left-right dimension. ${ }^{16}$ By combining the NPAT measures with campaign contribution

\footnotetext{
${ }^{15}$ For information on the NPAT data, see http://www.vote-smart.org

${ }^{16}$ See Ansolabahere et al. (2001) for a method for scaling the NPAT responses into ideology measures for incumbents and challengers.
} 
data drawn from the Federal Election Commission, it would be straightforward to analyze whether incumbents attract fewer funds in electorally and spatially close races. Alternatively (and closer to the results of the model), one could collect early polling data in congressional races to determine which candidate was the a priori favored candidate, and investigate whether that candidate received less money if the race was spatially close. While the model offers much in the way of indeterminacy, it does offer a sufficiently clear prediction, so that careful empirical analysis is possible. Future extensions to this project will aim to incorporate empirical approaches such as these, but for the moment, the model developed provides a more refined picture of possible donor and candidate behaviors that follow from the introduction of money into electoral competition.

\section{APPENDIX}

\section{Proof of Lemma 1}

The result is immediate as (7) involves the relevant expected utilities to the binary action facing a contributor with ideal point $x$ and the conjecture that $R$ characterizes the other contributor's behavior.

\section{Proof of Lemma 2}

To establish existence, note that by Definition 1 it is sufficient to find a function $z(R):[0,1] \rightarrow[0,1]$ s.t. $x \leq z(R)$ implies that $E u(x, 1, w) \leq$ $E u(x, 0, w)$ and $x \geq z(R)$ implies $E u(x, 1, w) \geq E u(x, 0, w)$ (because, given such a function, the set $b^{-1}(1)$ is just $\left.\left\{(x, R) \in[0,1]^{2}: x \geq z(R)\right\}\right)$. From Lemma 1 it must be the case that $x \geq z(R)$ iff

$$
\left|x-c_{0}\right|-\left|x-c_{1}\right| \geq\left(\frac{1-\kappa}{\kappa}\right)(1-2 p(R)) .
$$

For $x \in\left[c_{0}, c_{1}\right]$ this condition is equivalent to

$$
x \geq\left(\frac{1-\kappa}{\kappa}\right)\left(\frac{1}{2}-p(R)\right)+\frac{c_{0}+c_{1}}{2} .
$$

For $x \leq c_{0}$ this condition is equivalent to

$$
\frac{c_{0}-c_{1}}{2} \geq\left(\frac{1-\kappa}{\kappa}\right)\left(\frac{1}{2}-p(R)\right) .
$$

For $x>c_{1}$ this condition is equivalent to

$$
\frac{c_{1}-c_{0}}{2} \geq\left(\frac{1-\kappa}{\kappa}\right)\left(\frac{1}{2}-p(R)\right) \text {. }
$$


Consider three cases: First, assume

$$
\left(\frac{1-\kappa}{\kappa}\right)\left(\frac{1}{2}-p(R)\right)+\frac{c_{0}+c_{1}}{2} \in\left[c_{0}, c_{1}\right]
$$

If $x \geq c_{1}$ then

$$
c_{1}>\left(\frac{1-\kappa}{\kappa}\right)\left(\frac{1}{2}-p(R)\right)+\frac{c_{0}+c_{1}}{2}
$$

and thus (18) is satisfied. If $x \leq c_{0}$ then

$$
c_{0}<\left(\frac{1-\kappa}{\kappa}\right)\left(\frac{1}{2}-p(R)\right)+\frac{c_{0}+c_{1}}{2}
$$

and thus

$$
\frac{c_{0}-c_{1}}{2}<\left(\frac{1-\kappa}{\kappa}\right)\left(\frac{1}{2}-p(R)\right)
$$

so that (17) is not satisfied. Accordingly if

$$
\left(\frac{1-\kappa}{\kappa}\right)\left(\frac{1}{2}-p(R)\right)+\frac{c_{0}+c_{1}}{2} \in\left[c_{0}, c_{1}\right]
$$

then

$$
x \geq\left(\frac{1-\kappa}{\kappa}\right)\left(\frac{1}{2}-p(R)\right)+\frac{c_{0}+c_{1}}{2} \in\left[c_{0}, c_{1}\right]
$$

is necessary and sufficient for contribution to candidate 1 to be a best response. Second, assume

$$
\left(\frac{1-\kappa}{\kappa}\right)\left(\frac{1}{2}-p(R)\right)+\frac{c_{0}+c_{1}}{2}<c_{0}
$$

then it must be the case that $p(R)>\frac{1}{2}$ and thus (18) is true. Moreover

$$
\left(\frac{1-\kappa}{\kappa}\right)\left(\frac{1}{2}-p(R)\right)+\frac{c_{0}+c_{1}}{2}<c_{0}
$$

implies that

$$
\left(\frac{1-\kappa}{\kappa}\right)\left(\frac{1}{2}-p(R)\right)<\frac{c_{0}-c_{1}}{2}
$$

and thus (17) is true. Accordingly if

$$
\left(\frac{1-\kappa}{\kappa}\right)\left(\frac{1}{2}-p(R)\right)+\frac{c_{0}+c_{1}}{2}<c_{0}
$$


then contribution to 1 is a best response for all types of contributors. Third, assume

$$
\left(\frac{1-\kappa}{\kappa}\right)\left(\frac{1}{2}-p(R)\right)+\frac{c_{0}+c_{1}}{2}>c_{1}
$$

In this case $p(R)<\frac{1}{2}$ implying that the right-hand side of (17) is positive while the left-hand side is negative and thus (17) cannot be true. Moreover, if

$$
\left(\frac{1-\kappa}{\kappa}\right)\left(\frac{1}{2}-p(R)\right)+\frac{c_{0}+c_{1}}{2}>c_{1}
$$

then

$$
\left(\frac{1-\kappa}{\kappa}\right)\left(\frac{1}{2}-p(R)\right)>\frac{c_{1}-c_{0}}{2}
$$

implying that (18) is not true. Accordingly, if

$$
\left(\frac{1-\kappa}{\kappa}\right)\left(\frac{1}{2}-p(R)\right)+\frac{c_{0}+c_{1}}{2}>c_{1}
$$

then contribution to 0 is a best response for all types of contributors. Given this, the function (8) gives best responses. This establishes existence. Note that since $p(R)$ is increasing, $z(R)$ is non-increasing in $R$ and the graphs of the sets $\{(x, R): x<z(R)\},\{(x, R): x>z(R)\}$ are connected sets. Hence, the sets $b(1)^{-1}$ and $b(0)^{-1}$ are connected.

\section{Proof of Proposition 1}

Note that by Lemma 2, a profile of simultaneous best responses exists. It is therefore sufficient to establish the existence of a fixed point to the mapping $1-F(z(\cdot)):[0,1] \rightarrow[0,1]$. By inspection of equation (8) the function $z(p(R))$ is non-increasing in $p(R)$, and $p(\cdot)$ is non-decreasing by assumption. Therefore, $z(R)$ is non-increasing in $R$. Since $F(\cdot)$ is non-decreasing, $1-F(z(\cdot)):[0,1] \rightarrow[0,1]$ is a non-decreasing function from $[0,1]$ into itself. Hence, by Tarsky's fixed-point theorem an $R^{*}$ satisfying (9) exists.

\section{Proof of Proposition 2}

To prove (i), note that $R^{*}=1$ if and only if $1-F(z(1))=1$. Since $F(\cdot)$ is strictly increasing and $F(0)=0$ this means $R^{*}=1$ if and only if $z(1) \leq 0$. This inequality is true if and only if

$$
p(1) \geq \frac{\kappa}{1-\kappa}\left(\frac{c_{1}-c_{0}}{2}\right)+\frac{1}{2} .
$$

To prove (ii), note that $R^{*}=0$ if and only if $1-F(z(0))=0$. Since $F(\cdot)$ is strictly increasing and $F(1)=1$ this means $R^{*}=0$ if and only if $z(0) \geq 1$. This 
inequality is true if and only if

$$
p(0) \leq \frac{\kappa}{1-\kappa}\left(\frac{c_{0}-c_{1}}{2}\right)+\frac{1}{2} .
$$

\section{Proof of Proposition 3}

Since $1-F(z(R))$ is weakly increasing and $p(\cdot)$ is weakly increasing it is sufficient to consider the cases of $p(0)=0$ and $p(1)=1$. In the former case $\xi(1)>0$ if

$$
1<\frac{\kappa}{1-\kappa}\left(\frac{c_{1}-c_{0}}{2}\right)+\frac{1}{2} \text {. }
$$

In the latter case $\xi(0)<1$ if

$$
\frac{\kappa}{1-\kappa}\left(\frac{c_{0}-c_{1}}{2}\right)+\frac{1}{2}<0 .
$$

\section{Proof of Proposition 4}

Having a countable number of fixed points and having locally unique fixed points are equivalent conditions. In establishing this latter condition, it is sufficient to show that the set of parameter vectors upon which a continuum of equilibria exists has an empty interior. By way of contradiction, assume that for a parameter vector $\theta^{\prime}$, there exists an open ball of $\theta^{\prime}$ with radius $\varepsilon$, denoted $n b d\left(\theta^{\prime}, \varepsilon\right)$ upon which $\mathscr{R}^{*}(\theta) \cap(0,1)$ is uncountable. This implies that for some $R^{*} \in \mathscr{R}^{*}(\theta) \cap(0,1)$, the following conditions are true:

$$
\begin{aligned}
& R^{*}=1-F\left(z\left(R^{*} ; \theta\right)\right) \\
& \left.\frac{\partial F(z(R ; \theta))}{\partial R}\right|_{R^{*}}=-1
\end{aligned}
$$

for every $\theta \in n b d\left(\theta^{\prime}, \varepsilon\right)$. By the chain rule equation (20) is equivalent to

$$
-\frac{1}{p^{\prime}\left(R^{*}\right)}=\left(\frac{1-\kappa}{\kappa}\right) F^{\prime}\left(\left(\frac{1-\kappa}{\kappa}\right)\left(\frac{1}{2}-p(R)\right)+\frac{c_{0}+c_{1}}{2}\right) .
$$

But since the left-hand side is constant in $\theta$ and the right-hand side is strictly increasing in $c_{0}$ (21) cannot hold on $n b d\left(\theta^{\prime}, \varepsilon\right)$. Thus we have attained a contradiction, and the number of fixed points is countable. Since $F(\cdot)$ and $p(\cdot)$ are continuous on a compact domain, they are uniformly continuous; and thus having a countable number of fixed points implies having a finite number of fixed points, the result is established.

\section{Proof of Lemma 3}

By construction, $R^{*}$ solves the equation: $R^{*}-1+F\left(z\left(R^{*} ; \kappa\right)\right)=0$. At an interior solution satisfying a generic transversality condition 
$(1 \neq \partial F(z(R ; \theta)) / \partial R)$, the implicit function theorem implies that the following identity holds:

$$
\frac{\partial R^{*}}{\partial \theta_{i}}=-\frac{\frac{\partial F(z(R ; \theta))}{\partial \theta_{i}}}{1+\frac{\partial F(z(R ; \theta))}{\partial R}} .
$$

Calculation of $\partial F(z(R ; \theta)) / \partial R$ indicates that the sign of the left-hand side is the opposite of (same as) the sign of the numerator if

$$
F^{\prime}(z(R))\left(\frac{1-\kappa}{\kappa}\right) p^{\prime}(R)>(<) 1 \text {. }
$$

\section{Proof of Proposition 5}

Assume $R^{*} \in U(\theta)$. By Lemma 3, $\partial R^{*} / \partial \kappa$ has the opposite sign of $\partial F(z(R ; \theta)) / \partial \kappa$. By the chain rule this term is:

$$
\frac{\partial F(z(R ; \theta))}{\partial \kappa}=\left(\frac{\partial F(z(R ; \theta))}{\partial z}\right)\left(\frac{\partial z(R ; \theta)}{\partial \kappa}\right)
$$

and by equation (8):

$$
\left(\frac{\partial z(R ; \theta)}{\partial \kappa}\right)=\left(\frac{1}{2}-p(R)\right)\left(\frac{-1}{\kappa^{2}}\right)
$$

which is positive if $p\left(R^{*}\right)>\frac{1}{2}$ and negative if $p\left(R^{*}\right)<\frac{1}{2}$. Since $F(\cdot)$ is strictly increasing, Proposition 5 must be true.

\section{Proof of Proposition 6}

(i) Assume $R^{*} \in U(\theta)$. By Lemma 3, $\partial R^{*} / \partial c_{j}$ has the opposite sign of $\partial F(z(R ; \theta)) / \partial c_{j}$. By the chain rule then, this term is:

$$
\frac{\partial F(z(R ; \kappa))}{\partial c_{j}}=\frac{F^{\prime}(R)}{2},
$$

which is positive. Thus, the claim is established.

\section{Proof of Proposition 7}

Assume $c_{0}=c_{1}$. It follows then that $z(R)=0(1)$ if $p(R)>(<) \frac{1}{2}$. If $p(R)=\frac{1}{2}$ we assume that contributors flip a coin. Thus, equation (9) becomes

$$
R= \begin{cases}1 & \text { if } p(R)>\frac{1}{2} \\ R & \text { if } p(R)=\frac{1}{2} \\ 0 & \text { otherwise }\end{cases}
$$


To establish part (1) note that since $p(\cdot)$ is strictly increasing, at least one of the following conditions hold: (i) $p(0) \leq \frac{1}{2}$ or (ii) $p(1) \geq \frac{1}{2}$. In the former, $0 \in \mathscr{R}$, and in the latter, $1 \in \mathscr{R}$. To establish (2) note that if $p(R)=\frac{1}{2}$ then $R \in \mathscr{R}$. It is clear that if $p\left(\frac{1}{2}\right)=\frac{1}{2}$ then $R^{*} \in\left\{0, \frac{1}{2}, 1\right\}$ solves (9). To establish (3) assume that an interior $R^{*}$ exists. Note that if $p\left(\frac{1}{2}\right)<(>) \frac{1}{2}$ then by the monotonicity of $p(\cdot)$ at $R^{*} \leq(\geq) \frac{1}{2}$ it must be true that we have $p\left(R^{*}\right)<(>) \frac{1}{2}$. (But) since an interior solution requires $p\left(R^{*}\right)=\frac{1}{2}$, however, it must be true that $R^{*}<(>) \frac{1}{2}$.

\section{Proof of Lemma 4}

For a fixed mapping, the equivalent game in which candidates simultaneously choose $c_{0}, c_{1}$ and have payoffs $-\int P(R) d G\left(R ; c_{0}, c_{1}\right)$ and $\int P(R) d G\left(R ; c_{0}, c_{1}\right)$ respectively is a zero-sum game and it is well known that all Nash equilibria to this game are payoff equivalent (see, for example, Osbourne and Rubinstein, 1994, proposition 22.2). Since $G\left(\cdot ; c_{0}, c_{1}\right),\left(c_{0}^{*}, c_{1}^{*}\right)$ is a CCPE iff $\left(c_{0}^{*}, c_{1}^{*}\right)$ is a Nash equilibrium to the equivalent game. The result is established.

\section{Proof of Proposition 8}

It is sufficient to construct a common belief mapping $G\left(\cdot ; c_{0}, c_{1}\right)$ for which $c_{0}=c_{1}=m$ solves (14). Let

$$
G\left(R ; c_{0}, c_{1}\right)= \begin{cases}1 & \text { if } R=p^{-1}\left(\frac{1}{2}\right) \text { and } c_{0}=c_{1} \\ 1 & \text { if } R=\inf \left\{\mathscr{R}^{*}\left(c_{0}, c_{1}\right)\right\} \text { and } 0<\left|c_{0}-m\right| \leq\left|c_{1}-m\right| \\ 1 & \text { if } R=\sup \left\{\mathscr{R}^{*}\left(c_{0}, c_{1}\right)\right\} \text { and }\left|c_{0}-m\right|>\left|c_{1}-m\right| \\ 0 & \text { otherwise. }\end{cases}
$$

Clearly, if (i) $p\left(\inf \left\{\mathscr{R}^{*}\left(m, c_{1}\right)\right\}\right) \leq \frac{1}{2}$ for all $c_{1}$ and (ii) $p\left(\sup \left\{\mathscr{R}^{*}\left(c_{0}, m\right)\right\}\right) \geq$ $\frac{1}{2}$ for all $c_{0}$ then a unilateral deviation from $c_{0}=c_{1}=m$ (which results in payoffs of $\frac{1}{2}$ with probability 1 for each player) is not desirable. To show (i) augment the notation so that

$$
\xi\left(R, c_{0}, c_{1}\right)=\left(\frac{1-\kappa}{\kappa}\right)\left(\frac{1}{2}-p(R)\right)+\frac{c_{0}+c_{1}}{2} .
$$

Consider an arbitrary $c_{1}>m$. If

$$
p(0) \leq \frac{\kappa}{1-\kappa}\left(\frac{m-c_{1}}{2}\right)+\frac{1}{2}
$$

then $0=\inf \left\{\mathscr{R}^{*}\left(m, c_{1}\right)\right\}$ and the unilateral deviation from $m$ to $c_{1}$ is undesirable establishing (i). If instead we have

$$
p(0)>\frac{\kappa}{1-\kappa}\left(\frac{m-c_{1}}{2}\right)+\frac{1}{2}
$$


then since $p(\cdot)$ is non-decreasing it is the case that

$$
\left(\frac{1-\kappa}{\kappa}\right)\left(\frac{1}{2}-p(R)\right)<\frac{c_{1}-m}{2}
$$

Adding $-c_{1}$ to both sides yields

$$
\left(\frac{1-\kappa}{\kappa}\right)\left(\frac{1}{2}-p(R)\right)+\frac{c_{1}+m}{2}<c_{1}
$$

which implies that $z\left(R ; m, c_{1}\right)$ is continuous in $R$ for $R<1$. This implies that $\inf \left\{\mathscr{R}^{*}\left(m, c_{1}\right)\right\}$ is an upper crossing fixed point. To establish (i) we show that for every $\varepsilon \geq m$ the function $\inf \left\{\mathscr{R}^{*}(m, \varepsilon+\delta)\right\}$ is weakly decreasing in $\delta$ on some neighborhood of 0 (i.e. for $\delta$ sufficiently small). This observation, the monotonicity of $p(\cdot)$ and (A2), implies that if $c_{1}>m$ then $p\left(\inf \left\{\mathscr{R}^{*}\left(m, c_{1}\right)\right\}\right) \leq \frac{1}{2}$. Similar arguments handle the inverted case of $c_{1}<m$. Augmenting the notation to denote $z\left(R ;\left(c_{0}+c_{1}\right) / 2\right)$ we have the identity

$$
\left\{\mathscr{R}^{*}(m, \varepsilon+\delta)\right\}:=\left\{R: R=1-F\left(z\left(R ; \frac{\varepsilon+\delta}{2}\right)\right) .\right.
$$

Since $\inf \left\{\mathscr{R}^{*}(m, \varepsilon+\delta)\right\}$ is an upper crossing fixed point, Proposition $6 \mathrm{im}-$ plies that $\inf \left\{\mathscr{R}^{*}(m, \varepsilon+\delta)\right\}$ is decreasing in $\delta$ on a neighborhood of 0 if the derivative exists. If the transversality condition is satisfied at $\inf \left\{\mathscr{R}^{*}(m, \varepsilon)\right\}$ Proposition 6 implies that $\inf \left\{\mathscr{R}^{*}(m, \varepsilon+\delta)\right\}$ is decreasing in $\delta$ on a neighborhood of 0 . If the transversality condition is not satisfied at $\inf \left\{\mathscr{R}^{*}(m, \varepsilon)\right\}$ then there is a discontinuity in $\inf \left\{\mathscr{R}^{*}(m, \varepsilon)\right\}$. But since $\inf \left\{\mathscr{R}^{*}(m, \varepsilon)\right\}$ is an upper crossing we know that in this case $\lim _{\delta \uparrow 0} \inf \left\{\mathscr{R}^{*}(m, \varepsilon+\delta)\right\}$ $<\inf \left\{\mathscr{R}^{*}(m, \varepsilon)\right\}$, so $\inf \left\{\mathscr{R}^{*}(m, \varepsilon+\delta)\right\}$ is decreasing in $\delta$ at $(m, \varepsilon)$.

To establish (ii) a virtually equivalent argument can be constructed using the fact that $\sup \left\{\mathscr{R}^{*}\left(c_{0}, m\right)\right\}$ is either an upper crossing fixed point or equal to 1 . Hence, $\sup \left\{\mathscr{R}^{*}(\varepsilon+\delta, m)\right\}$ is decreasing in $\delta$ at neighborhoods of 0 for every $\varepsilon<m$. This and (A3) yield the result.

\section{Proof of Proposition 9}

Assume that $c_{0}, c_{1}$ solves (14) given (27). By Lemma 4, we know that in every CCPE involving the mapping in (27) the equilibrium probability that candidate 1 wins is $\frac{1}{2}$. Hence, it must be true that if $\left(c_{0}+c_{1}\right) / 2 \neq m$, then

$$
p^{-1}\left(\frac{1}{2}\right)=1-F\left(z\left(R ; \frac{c_{0}+c_{1}}{2}\right)\right) .
$$

So, if $p^{-1}\left(\frac{1}{2}\right)<(>)(=) \frac{1}{2}$, then $\left(c_{0}+c_{1}\right) / 2>(<)(=) m$. In the first case we have $\left|c_{0}-m\right| \leq\left|c_{1}-m\right|$ and (27) implies that $R=\inf \left\{\mathscr{R}^{*}\left(c_{0}, c_{1}\right)\right\}$. By Proposition 6, however, for $\lambda>0$ (small), the deviation to $\lambda m+(1-\lambda) c_{1}$ is desirable for 1 unless $R=\inf \left\{\mathscr{R}^{*}\left(c_{0}, c_{1}\right)\right\}=0$. So either $R=0$ in equilibrium or 1 has an incentive to deviate. But $p^{-1}\left(\frac{1}{2}\right)=0$ cannot attain given 
(A1). If $p^{-1}\left(\frac{1}{2}\right)>\frac{1}{2}$ then a similar argument implies that 0 would have an incentive to deviate from $c_{0}$. If $p^{-1}\left(\frac{1}{2}\right)=\frac{1}{2}$ then $\left(c_{0}+c_{1}\right) / 2=m$ and (27) indicates that $R=1-F(m)=\frac{1}{2}$. But by Proposition 6 for $\lambda>0$, (small) the deviation to $\lambda m+(1-\lambda) c_{0}$ is desirable for 0 . Thus we have attained the contradiction.

\section{Proof of Proposition 10}

Assume $p(0)=0, p(1)=1$, and $\kappa \leq \frac{1}{2}$. By Proposition 2(i), $R^{*}=1$ is an equilibrium if

$$
1 \geq \frac{\kappa}{1-\kappa}\left(\frac{c_{1}-c_{0}}{2}\right)+\frac{1}{2}
$$

Since $c_{1}-c_{0} \leq 1,1$ is an equilibrium proportion to the contribution game if $\kappa \leq \frac{1}{2}$. By Proposition 2(ii), $R^{*}=0$ is an equilibrium proportion if and only if

$$
0 \leq \frac{\kappa}{1-\kappa}\left(\frac{c_{0}-c_{1}}{2}\right)+\frac{1}{2} .
$$

Since $c_{0}-c_{1} \geq-1,0$ is an equilibrium proportion to the contribution game if $\kappa \leq \frac{1}{2}$. This implies that for any $\left(c_{0}, c_{1}\right),\{0,1\} \subset \mathscr{R}\left(c_{0}, c_{1}\right)$. Since we have assumed that $p(0)=0$ and $p(1)=1$, it must be true that for any function $\psi:[0,1]^{2} \rightarrow[0,1]$, we can have $\int P(R) d G\left(R ; c_{0}, c\right)=\psi\left(c_{0}, c\right)$ by setting $G\left(1 ; c_{0}, c_{1}\right)=\psi\left(c_{0}, c_{1}\right)$ and $G\left(0 ; c_{0}, c_{1}\right)=1-\psi\left(c_{0}, c_{1}\right)$. Thus, it is sufficient to show that for any $\left(c_{0}^{\prime}, c_{1}^{\prime}\right) \in[0,1]^{2}$ there is some function $\psi:[0,1]^{2} \rightarrow[0,1]$ for which:

$$
\begin{aligned}
& c_{1}^{\prime} \in \arg \max _{c \in[0,1]}\left\{\psi\left(c_{0}^{\prime}, c\right)\right\} \\
& c_{0}^{\prime} \in \arg \max _{c \in[0,1]}\left\{1-\psi\left(c, c_{1}^{\prime}\right)\right\} .
\end{aligned}
$$

For any $\left(c_{0}^{\prime}, c_{1}^{\prime}\right) \in[0,1]^{2}$ the following is such a function, $\psi\left(c_{0}, c_{1}\right)=a$ for some $a \in[0,1]$. Of course a function which makes these optima unique can also be constructed.

\section{ACKNOWLEDGMENTS}

The authors wish to thank Jeffrey Lewis, John Patty, Kenneth Shotts, and anonymous reviewers for insightful comments. An earlier version of this paper was presented at the Annual Meeting of the American Political Science Association.

ADAM MEIROWITZ

ALAN E. WISEMAN

Department of Politics,

Princeton University

Department of Political Science, The Ohio State University 
Ansolabehere, S. D., J. M. Snyder Jr., and C. Stewart III, 2001, Candidate positioning in US House elections. American Journal of Political Science 1, 136-159.

Baron, D. P., 1994, Spatial competition and campaign contributions with informed and uninformed voters. American Political Science Review 88, 33-47.

Brown, C. W., Jr., L. W. Powell, and C. Wilcox, 1995, Serious Money, Fund-Raising and Contributing in Presidential Nomination Campaigns (Cambridge University Press, Cambridge, UK).

Dixit, A., 1986, Comparative statics for oligopoly. International Economic Review 27, $107-122$.

Downs, A., 1957, An Economic Theory of Democracy (Harper \& Row, New York).

Dunn, D. D., 1972, Financing Presidential Campaigns (Brookings Institution Press, Washington, DC).

Economides, N., 1996, The economics of networks. International Journal of Industrial Organization 14, 673-699.

Farrell, J. and G. Saloner, 1985, Standardization, compatibility, and innovation. Rand Journal of Economics 16, 70-83.

- and - 1986 , Installed base and compatibility: innovation, product preannouncements, and predation. American Economic Review 76, 940-955.

Fisher, F., 1961, The stability of the Cournot oligopoly solution: the effects of speed of adjustment and increasing marginal costs. Review of Economic Studies $28,125-135$.

Francia, P. L., R. E. Goldberg, J. C. Green, P. S. Herrnson, and C. Wilcox, 1999, Individual donors in the 1996 Federal elections, in: J. C. Green, ed., Financing the 1996 Election (M. E. Sharpe, Armonk, NY).

Fudenberg, D. and J. Tirole, 1991, Game Theory (MIT Press, Cambridge, MA).

Glasser, S. B., 1999, Quayle leaves the stage but pledges to work on uniting GOP. The Washington Post, September 28, p. A10.

Hahn, F., 1962, The stability of the Cournot oligopoly solution. Review of Economic Studies 29, 929-931.

Jacobson, G. C., 1980, Money in Congressional Elections (Yale University Press, New Haven, CT).

—_, 1992, The Politics of Congressional Elections, 3rd edn. (HarperCollins, New York).

Judd, K., 1985, The law of large numbers with continuum of IID random variables. Journal of Economic Theory 35, 19-25.

Katz, M. L. and C. Shapiro, 1985, Network externalities, competition, and compatibility. American Economic Review 75, 424-440.

—_ and — 1986, Technology adoption in the presence of network externalities. Journal of Political Economy 94, 822-841.

- and -1994 , Systems competition and network effects. Journal of Economic Perspectives 8, 93-115.

Miga, A., 1999, Funds lacking, Dole bows out of prez race. The Boston Herald, October 21.

Morton, R. B. and R. B. Myerson, 2000, Decisiveness of contributors' perceptions in elections. Typescript, University of Houston.

Osbourne, M. J. and A. Rubinstein, 1994, A Course in Game Theory (MIT Press, Cambridge, MA).

Rohlfs, J., 1974, A theory of interdependent demand for a communications service. Bell Journal of Economics 5, 16-37. 
Snyder, J. M., 1990, Campaign contributions as investments. Journal of Political Economy 98, 1195-1227.

Sorauf, F. J., 1988, Money in American Elections (Scott, Foresman \& Co., Boston, MA).

, 1992, Inside Campaign Finance (Yale University Press, New Haven, CT).

Van Natta, D., Jr., 2000, Early rush of contributions opened floodgates for Bush. The New York Times, January 29.

Wilkie, D., 1999, Death of a political campaign: latest victim classic example. The San Diego Union-Tribune, October 21. 